\title{
Pricing a Lease Contract in Presence of Late Payment Extra-Charges
}

\author{
Luciano Quattrocchio ${ }^{1}$, Luisa Tibiletti ${ }^{1} \&$ Mariacristina Uberti $^{1}$ \\ ${ }^{1}$ School of Management and Economics, Department of Management, University of Torino, Torino, Italy \\ Correspondence: Mariacristina Uberti, School of Management and Economics, Department of Management, \\ University of Torino, C.so Unione Sovietica 218 bis, 10134 Torino, Italy. E-mail: mariacristina.uberti@unito.it
}

Received: July 25, 2019

doi:10.5539/ijbm.v14n11p179
Accepted: September 19, 2019

Online Published: October 19, 2019

URL: https://doi.org/10.5539/ijbm.v14n11p179

\begin{abstract}
Partial insolvency in leasing contracts may entail to afford additional late payment costs. In this paper we focus on the case that the lessee makes partial payments in due time and settles the debt augumented by the late payment interests later. The presence of the extra-costs drives the lease Effective Annual interest Rate $(E A R)$ to deviate from the lease contract rate. The aim of this work is to illustrate how design the contract payback amortization to stick $E A R$ to the lease contract rate, when the lease contract rate, the late payment rate and the contract term are exogeneously fixed. First we achieve a proxy for $E A R$ given by the lease contract rate plus an extra-charge rate addendum. We show that this latter addendum is sensitive to the payback Macaulay Duration, a weighted size and timing average. Specifically, the longer the Macaulay Duration, the smaller the extra-charge rate addendum. As a consequence, two general rules to drive $E A R$ close to the lease contract rate roll out, specifically: (1) the payment pattern should be set with a long Macaulay Duration; and (2) the surrender value of the leased good should be put large. As the contract settlement is given, we show that EAR is delimited by a lower bound and an upper bound. Then the payback amortizations with fixed instalments are studied. To get insight on the importance of $E A R$ inputs we roll sensitivity analysis out through illustrative applications. The results of the paper are useful to provide policymakers a better knowledge about the effects on EAR of the contract conditions on the pattern of payments.
\end{abstract}

Keywords: consumer credit, Effective Annual interest Rate (EAR), late payment fees, Macaulay duration, partial insolvency, policy

\section{Introduction}

An evergreen issue in designing leasing contract conditions is to keep the Effective Annual interest Rate $(E A R)$ under control in the presence of extra-costs. In recent times the question has become even more pressing due to a growing number of insolvencies of payments by debtors. Consequently, the need to monitor the cost of credit plays a significant role so that it is kept below some interest rate caps on loans imposed also by the legislation of various countries (see Maimbo \& Gallegos, 2014, Migliavacca et al., 2018). The main reason for using interest caps on loans is to protect consumers from excessive interest rates, to increase access to finance and to make loans more affordable.

Within this policy framework, this article analyses the extra-costs effects on $E A R$ when the lessee is partially insolvent and the contract interest rate, the late payment rate and the contract term are exogeneously fixed at the beginning of the contract. We get insights on the link between the cashflow pattern and the EAR under lessee's partial insolvency. Our main result concerns to show that the Macaulay Duration (Macaulay, 1938) a weighted size and timing average, can be used as a worthwhile sensitivity measure of the effects of extra-costs on EAR.

Designing leasing contract conditions that allow to move $E A R$ - all else being equal - is a key issue in developing contract conditions on the pattern of payments (see Carretta \& Nicolini, 2009; Quattrocchio et al., 2018). A general rule comes out: to stick EAR to the contract lease rate the Macaulay Duration should be set long.

We also carry out a number of simulations to assess the robustness of our results and to stress test for different types of patterns of contractual payments characterized by different Macaulay Durations.

The remainder of the paper is organized as follows. In Section 2, the model is set up. In Section 3 we study the impact on EAR of the payment payback settlement. In Section 4 we study the case of the payment of fixed payments and the possible option of the payment of the surrender value at the contract terminal date. Section 5 presents our results through simulations and discusses the outcomes of several sensitivity checks. Section 6 
concludes the note. The proofs are collected in the Appendix.

\section{Notation and Layout of the Model}

We lay out the notation used throughout the paper. Let

- $A$ be the leased amount, that is to be intended as the sum of the good price plus the initial costs needed to settle the contract;

- $\quad i$ be the annual compound lease contract rate;

- $\quad n$ be the number of installments of the contract. To explanatory purposes we assume that the installments are annually paid and so, $n$ coincides with the contract terminal date;

- $\quad R_{s} \geq 0$ be the payment due at the date $s$, with $s=1, \ldots, n$. Each $R_{s}$ is given by the sum of the capital share and the interest share;

- $E$ with $E \geq 0$ be the surrender value of the leased good to be paid at the date $n$ in addition to the last payment $R_{n}$ so that the payments $R_{s}, s=1, \ldots, n$, with $E$ payback the initial debt $A$

- $\quad \rho$, with $0 \leq \rho \leq 1$ be the fixed percentage of the instalment paid in due time;

- $\rho \cdot R_{s}$ be the amount paid at the date $s$, with $s=1, \ldots, n$;

- $\quad(1-\rho) \cdot R_{s}$ be the unpaid amount at the date $s$, with $s=1, \ldots, n$;

- $\quad m$ be the late payment annual interest rate. Usually the annual late payment interest rate is given as a nominal annual interest rate convertible the same number of times of the installments in a year. Without loss of generality, we can indicate with $m$ the periodical compound interest rate with the same period of installments dates where, in this paper, the period is the year.

We assume that contract expires at the date $n$, when the lessee pays the last installment plus the unpaid amounts augumented by the late payment interests and the surrender value if provided for by the contract.

The basic contract conditions are assumed to hold, see for example Brealey et al. (2014) and Yoshida et al. (2016, and the literature cited therein). We assume that the payments $R_{s}, s=1, \ldots, n$ are arbitrary chosen and solve the payback closure condition, i.e.

$$
A=\sum_{s=1}^{n} \frac{R_{s}}{(1+i)^{s}}+\frac{E}{(1+i)^{n}}
$$

Under the constraint (1) there are infinite different ways to settle the payment stream $R_{s}, s=1, \ldots, n$. In the following we will give a guide to select to proper payback amortization in order to move EAR toward an appropriate target value.

By definition the Net Present Value (NPV) at the lease contract rate $i$ of the contract is null

$$
N P V(i)=A-\sum_{s=1}^{n} \frac{R_{s}}{(1+i)^{s}}-\frac{E}{(1+i)^{n}}=0
$$

\section{Partial Payments in Fixed Percentage of the Due Amounts}

Let us assume that the lessee pays a fixed percentage $\rho$, with $0 \leq \rho \leq 1$ of the payment in due time. So the amount $\rho \cdot R_{s}$ with $s=1, \ldots, n$ is paid in due time. The unpaid amounts overcharged by the late payment interests are settled at the contract expiration date $n$.

The Discounted Cash Flow of the contract including the extra-costs caused by payment delays is given by the Net Present Value $\left(N P V_{\text {late }}\right)$

$$
\begin{aligned}
& N P V_{\text {late }}(x)=A-\rho \cdot \sum_{s=1}^{n} \frac{R_{s}}{(1+x)^{s}}-\frac{1-\rho}{(1+x)^{n}} \cdot \sum_{s=1}^{n} R_{s} \cdot(1+m)^{n-s}-\frac{E}{(1+x)^{n}}= \\
& =A-\rho \cdot \sum_{s=1}^{n} \frac{R_{s}}{(1+x)^{s}}-(1-\rho) \cdot\left(\frac{1+m}{1+x}\right)^{n} \cdot \sum_{s=1}^{n} \frac{R_{s}}{(1+m)^{s}}-\frac{E}{(1+x)^{n}}
\end{aligned}
$$

Where $x$ is the annual interest rate used for discounting. 
By definition the Effective Annual interest Rate (EAR) of the contract solves the following equation (Note 1)

$$
N P V_{\text {late }}(E A R)=0
$$

By construction $N P V_{\text {late }}(x)$ is a strictly increasing function in $x$. In the following we set conditions to sign the spread between $E A R$ and the contract lease rate.

\section{Remark 1. The impact of the late payment interest rate on $E A R$}

Let the contract payments $R_{s}$, with $s=1, \ldots, n$ be regularly paid in fixed percentage $\rho$, with $0 \leq \rho \leq 1$ and the outstanding debt overcharged with the late payment rate $m$ be settled through a lump-sum payment at the contract term $n$. Let the lease contract rate $i$, the late payment rate $m$ and the contract term $n$ be exogenously given. The sign of the spread between $E A R$ and the lease contract rate $i$ is driven by the late payment rate
a. if $m<i$ then $m<E A R<i$;
b. if $m=i$ then $E A R=i$;
c. if $i<m$ then $i<E A R<m$.

See Appendix A for the proof.

Remark 1 spotlights the impact of the late payment rate in defining EAR bounds. Under cheap late payment conditions, i.e. $m<i, E A R$ flaws lower than the contract lease rate; vice versa under expensive late payment costs, i.e. $m>i, E A R$ goes higher than the contract lease rate. If $m=i$ late payments have no effect on $E A R$.

\subsection{A Measure of the Spread between EAR and the Contract Lease Rate}

Now we are ready to formulate an $E A R$ proxy.

\section{Result 1. An "extra-charge rate addendum"}

A proxy of EAR is given by the sum of lease contract rate $i$ plus an "extra-charge rate addendum"

$$
E A R \cong i+\frac{-N P V_{\text {late }}(i)}{N P V_{\text {late }}^{\prime}(i)}=i+(1+i) \cdot(1-\rho) \cdot \frac{\left[M+E \cdot(1+i)^{-n}\right]-A}{\rho \cdot A \cdot D+(1-\rho) \cdot\left[M+E \cdot(1+i)^{-n}\right] \cdot n}
$$

where

$$
\text { - } \quad M=\left(\frac{1+m}{1+i}\right)^{n} \cdot \sum_{s=1}^{n} \frac{R_{s}}{(1+m)^{s}}
$$

is the present value at the lease contract rate $i$ of the lump-sum due at the contract term $n$ if no payments are made before;

$$
\text { - } D=\frac{\sum_{s=1}^{n} s \cdot R_{s} \cdot(1+i)^{-s}+n \cdot E \cdot(1+i)^{-n}}{\sum_{s=1}^{n} R_{s} \cdot(1+i)^{-s}+E \cdot(1+i)^{-n}}=\frac{\sum_{s=1}^{n} s \cdot R_{s} \cdot(1+i)^{-s}+n \cdot E \cdot(1+i)^{-n}}{A}, \text { with } 1 \leq D \leq n
$$

is the Macaulay Duration of the contract payback discounted at the lease contract rate $i$. The Macaulay Duration is a well-established and practical concept in finance literature (see Macaulay, 1938; de La Grandville, 2003) and permits to summarizes, in a single time number, the relevant characteristics of contract payments (size and timing).

The term

$$
\varepsilon=(1+i) \cdot(1-\rho) \cdot \frac{\left[M+E \cdot(1+i)^{-n}\right]-A}{\rho \cdot A \cdot D+(1-\rho) \cdot\left[M+E \cdot(1+i)^{-n}\right] \cdot n}
$$


is called the "extra-charge rate addendum". Clearly, if $\rho=1$ and payments $R_{s}$ with $s=1, \ldots, n$ are regularly paid, $\varepsilon=0$.

By Remark 1. the EAR proxy (3.1) can be re-written:

a. if $m<i$, then $m<E A R \cong i-(1+i) \cdot(1-\rho) \cdot\left|\frac{\left[M+E \cdot(1+i)^{-n}\right]-A}{\rho \cdot A \cdot D+(1-\rho) \cdot\left[M+E \cdot(1+i)^{-n}\right] \cdot n}\right|<i$

b. if $m=i$, then $E A R=i$;

c. if $i<m$, then $i<E A R \cong i+(1+i) \cdot(1-\rho) \cdot \frac{\left[M+E \cdot(1+i)^{-n}\right]-A}{\rho \cdot A \cdot D+(1-\rho) \cdot\left[M+E \cdot(1+i)^{-n}\right] \cdot n}<m$

where $|. .$.$| stands for the absolute value operator. See the proof in Appendix B.$

\subsection{The Impact of the Pattern of Payments on EAR}

The choice of the contract payback settlement plays a crucial role in the determining the late extra-charges. As intuition suggests if "small payments come first and large payments come later", in the event of partial insolvency, the late payment costs turn low because the late payment costs are computed for small amounts, although for long periods. Under this payback schedule, EAR is expected to be close to the contract lease rate $i$.

Vice versa if "large payments come first and small payments come later", the late payment costs are expected to be expensive, because the late payment costs are referred to large amounts and computed for long periods. In such circumstances $E A R$ is expected to be significantly divergent from the contract lease rate $i$.

The Macaulay Duration $D$ of the contract payback, where $1 \leq D \leq n$ allows to formalize the above intuitive reasoning. $D$ achieves its minimum and maximum when the payment streams are concentrated in a lump-sum at the extreme dates, more precisely:

- the minimum $D_{\min }=\frac{R_{1} \cdot(1+i)^{-1}+n \cdot E \cdot(1+i)^{-n}}{A}$ is achieved if the payment payback provides a lump-sum at the time 1, i.e. the payment stream is $R_{1}=A \cdot(1+i)-E \cdot(1+i)^{-n+1}, R_{2}=0, \ldots, R_{n}=0$ and the surrender value $E$ payment at the time $n$; and

- the maximum $D_{\max }=n$ is achieved if contractual payback provides the contract payment stream

$$
R_{1}=0, R_{2}=0, \ldots, R_{n-1}=0 \text { and } R_{n}=A \cdot(1+i)^{n}-E \text { and the surrender value } E \text { payment at time } n . .
$$

By properly choosing the payback schedule we can drive $E A R$ in (3.1) to approach or diverge from the contract lease rate.

\section{Result 2. The Macaulay Duration as a sensitivity measure of the lease contract rate and EAR spread}

Let the contract period $n$ be fixed and the contract payments $R_{s}$, with $s=1, \ldots, n$ and the surrender value $E$ satisfy the closure condition (1), then

- the more "the small payments come first and the large payments come later", the more the Macaulay Duration $D$ goes long and the more EAR approaches to the contract rate $i$;

- the more "the large payments come first and the small payments come later", the more Macaulay Duration $D$ goes short and the more $E A R$ diverges from the contract rate $i$.

See Appendix C.1 and C.2 for the proofs.

A way to complain the condition "the small payments come first and the large payments come later" is to provide the payment stream $R_{s}$ with $s=1, \ldots, n$ increasing in size. A further strategy is to provide for a large surrender value $E$ to be paid at the date $n$. In fact, a large payment at the terminal date $n$ reduces the payments 
$R_{s}$, with $s=1, \ldots, n$ and it pushes the Macaulay Duration toward $D_{\max }=n$. Vice versa, to complain the condition "the large payments come first and the small payments come later" the payments $R_{s}$ with $s=1, \ldots, n$ should be set decreasing in amount and the surrender value $E$ should be set small.

Now we are ready to identify a lower bound and an upper bound for EAR. By (3.1) the maximum divergence of $E A R$ from the contract rate $i$ occurs when the absolute value of the extra-change addendum $|\varepsilon|$ is maximum. The extreme values for $E A R$ occur if the contract payments $R_{s}$ with $s=1, \ldots, n$ are settled to minimize the Macaulay Duration D, i.e. $D_{\min }=\frac{R_{1} \cdot(1+i)^{-1}+n \cdot E \cdot(1+i)^{-n}}{A}$ (see Result 2) therefore the payment stream is $R_{1}=A \cdot(1+i)-E \cdot(1+i)^{-n+1}, \quad R_{2}=0, \ldots, R_{n}=0$ and $E$ is paid at time $n$. The extreme $E A R$ value, denoted by $A P R_{D_{\min }}$ solves the equation

$$
A-\frac{\rho \cdot\left[A \cdot(1+i)-E \cdot(1+i)^{-n+1}\right]}{1+x}-\frac{(1-\rho) \cdot\left[A \cdot(1+i)-E \cdot(1+i)^{-n+1}\right] \cdot(1+m)^{n-1}}{(1+x)^{n}}-\frac{E}{(1+x)^{n}}=0
$$

\section{Result 3. A lower bound and an upper bound for $E A R$}

Let the contract payments $R_{s}$, with $s=1, \ldots, n$ be regularly paid in fixed percentage $\rho$ with $0 \leq \rho \leq 1$ and the outstanding debt overcharged, the late payment extra-costs and the surrender value $E$ settled at the contract term $n$. Assume that the lease contract rate $i$, the late payment rate $m$ and the contract term $n$ be exogenously given:

a. if $m<i$ then $\min E A R=E A R_{D_{\min }}$, that is approximated by $\min E A R \cong i-\left|\varepsilon_{D_{\min }}\right|$. The minimum $E A R$ is obtained because of taking advantage of the cheap late payment extra-costs. This advantage is emphasized by $D_{\min }$ such that large unpaid payments are settled at cheap late payment costs at date $n$

$$
\min E A R=E A R_{D_{\min }} \leq E A R \leq \max E A R=E A R_{D=n}=i ;
$$

b. if $m=i$ then $E A R=i$;

c. if $i<m$ then $\max E A R=E A R_{D_{\min }}$ that is approximated by $\max E A R \cong i+\varepsilon_{D_{\min }}$. The maximum $E A R$ is due to the expensive late payment costs which are settled at expensive late payment costs at date $n$

$$
\min E A R=E A R_{D=n}=i \leq E A R \leq \max E A R=E A R_{D_{\min }}
$$

The proof follows from Result 2 .

\subsection{Strategies to Design Leasing Contract Conditions to Meet the Appropriate EAR Target}

Designing leasing contract conditions that allow to move EAR toward an appropriate target is a key issue in developing contract conditions.

Cheap late payment charges (i.e. $m<i$ ) may incentive the lessee to delay payments; whereas expensive late payment extra-costs (i.e. $m>i$ ) may induce the lessee to turn down the contract. Therefore, if $m<i$ we expect that the policyholder be wishing to design contract conditions able to push $E A R$ up; vice versa, if $m>i$ to push $E A R$ down. In the both cases, the policyholder is expected to be willing to set $E A R$ as close as possible to the contract rate $i$. 


\section{Result 4. How designing contracts to move $E A R$ close to the contract rate}

Let the contract payments $R_{s}$ with $s=1, \ldots, n$ be regularly paid in fixed percentage $\rho$ with $0 \leq \rho \leq 1$ and the outstanding debt overcharged with the late payment interests and the surrender value $E$ be settled through a lump-sum payment at the contract term $n$. Let the lease contract rate $i$, the late payment rate $m$ and the contract term $n$ be exogenously given. A strategy to reduce the absolute value $|\varepsilon|$ of the extra-charge addendum is to make the Macaulay Duration $D$ long.

If the contract expiration date $n$ is fixed, the only way to prolong the Macaulay Duration $D$ is to properly set the payment stream $R_{s}$ with $s=1, \ldots, n$ and the surrender value $E$. A sufficient way to achieve this target is

- to set the contract payments $R_{s}$ with $s=1, \ldots, n$ increasing in amount and,

- if the contract provides for the surrender value $E$, set this value large.

As it has been already discussed, $D_{\max }=n$ is achieved if contractual payback provides $R_{1}=0, R_{2}=0, \ldots, R_{n-1}=0, R_{n}=A \cdot(1+i)^{n}-E$ and at time $n$ it provides the surrender value $E$, therefore the outflow at time $n$ is of $A \cdot(1+i)^{n}$. Clearly in such circumstance, there is no insolvence, $E A R=i$ and $|\varepsilon|=0$.

\section{Fixed Installments}

Let the leased amount $A$ be paid back through the payment of fixed installments at the dates $s=1,2, . ., n$. We separately consider the option of a zero-price and positive-price surrender value.

\subsection{Zero-Price Surrender Value}

Let the surrender value be $E=0$. According to the French amortization, the fixed instalments $R_{1}=R_{2}, \ldots=R_{n}=R$ at the dates $s=1,2, . ., n$ are equal to

$$
R=\frac{A}{a_{\vec{n} \mid i}}
$$

where $a_{\bar{n} \mid i}=\frac{1-(1+i)^{-n}}{i}$ is the present value of a unitary ordinary annuity.

The Macaulay Duration of the contract payments is

$$
D_{E=0}=\frac{\sum_{s=1}^{n} s \cdot R \cdot(1+i)^{-s}}{\sum_{s=1}^{n} R \cdot(1+i)^{-s}}=\frac{\sum_{s=1}^{n} s \cdot(1+i)^{-s}}{\sum_{s=1}^{n}(1+i)^{-s}}=1+\frac{1}{i}-\frac{n}{(1+i)^{n}-1} \quad, \quad \text { with } 1 \leq D_{E=0}<1+\frac{1}{i}
$$

see Dierkes and Ortmann (2015). Formula (5) shows that $D_{E=0}$ is independent on the leased amount $A$ and the installment $R$. As a consequence, the Macaulay Duration $D_{E=0}$ is uniquely determinated by the contract interest rate $i$ and the contract term $n$.

\subsection{Positive-Price Surrender Value}

Let $E$ be the surrender value with $E \geq 0$. The fixed installment becomes

$$
R_{E}=\frac{A-E \cdot(1+i)^{-n}}{a_{\vec{n} \mid i}}=R-\frac{E}{s_{\bar{n} \mid i}}=\frac{A}{a_{\bar{n} \mid i}}-\frac{E}{s_{\bar{n} \mid i}}=\frac{A \cdot(1+i)^{n}}{S_{\bar{n} \mid i}}-\frac{E}{S_{\bar{n} \mid i}}=\frac{A \cdot(1+i)^{n}-E}{S_{\bar{n} \mid i}}
$$

where $R$ is the fixed payment in (6) due if $E=0$ and $s_{\bar{n} \mid i}$ is the final amount of an ordinary annuity. If the surrender value $E>0$ the periodical payments reduce, i.e. $R_{E}<R$.

The Macaulay Duration of an outflow including the surrender value $E$ is 


$$
D=\frac{\sum_{s=1}^{n} s \cdot R_{E} \cdot(1+i)^{-s}+n \cdot E \cdot(1+i)^{-n}}{A}=D_{E=0}+\frac{E \cdot(1+i)^{-n}}{A} \cdot\left(n-D_{E=0}\right) \quad \text { where } 1 \leq D_{E=0} \leq D \leq n
$$

where $D_{E=0}$ is defined in (5), see Appendix D for the proof.

It is worthwhile noting that the Macaulay Duration $D$ in (6) depends on the leased amount $A$ and the surrender value $E$.

Therefore,

- if $E=0$ then $D=D_{E=0}$ defined in (5); and

- if $E>0$ then $D>D_{E=0}$ and the larger the surrender value $E$, the longer the Macaulay Duration $D$.

We can rewrite the $N P V_{\text {late }}$ formula (2) with fixed installments $R_{E}$, surrender value $E$, with $E \geq 0$

$$
\begin{aligned}
& N P V_{\text {late }}(x)=A-\rho \cdot R_{E} \cdot \sum_{s=1}^{n} \frac{1}{(1+x)^{s}}-(1-\rho) \cdot\left(\frac{1+m}{1+x}\right)^{n} \cdot R_{E} \cdot \sum_{s=1}^{n} \frac{1}{(1+m)^{s}}-\frac{E}{(1+x)^{n}}= \\
& =A-R_{E} \cdot\left[\rho \cdot \sum_{s=1}^{n} \frac{1}{(1+x)^{s}}+(1-\rho) \cdot\left(\frac{1+m}{1+x}\right)^{n} \cdot \sum_{s=1}^{n} \frac{1}{(1+m)^{s}}\right]-\frac{E}{(1+x)^{n}}= \\
& =A-R_{E} \cdot\left[\rho \cdot a_{n \mid x}+(1-\rho) \cdot\left(\frac{1+m}{1+x}\right)^{n} \cdot a_{n \mid m}\right]-\frac{E}{(1+x)^{n}}
\end{aligned}
$$

The exact $E A R$ value solves the equation $N P V_{\text {late }}(x)=0$. A proxy of $E A R$ is given by formula (3.1) and follows

$$
E A R \cong i+(1-\rho) \cdot(1+i) \cdot \frac{R_{E} \cdot\left[\frac{s_{n \mid m}}{s_{n \mid i}}-1\right]}{\rho \cdot\left[R_{E}+\frac{E}{S_{n \mid i}}\right] \cdot D_{E}+(1-\rho) \cdot\left[R_{E} \cdot \frac{s_{n \mid m}}{S_{n \mid i}}+\frac{E}{S_{n \mid i}}\right] \cdot n} \text { with } E \geq 0
$$

where $s_{n \mid m}=(1+m)^{n} \cdot a_{n \mid m}$ and $s_{n \mid i}=(1+i)^{n} \cdot a_{n \mid i}$, see Appendix E for the proof.

We are ready now to illustrate the main strategies to drive $E A R$ close to the contract interest rate $i$.

\section{Result 5. Fixed payments: how designing contracts to move $E A R$ close to the contract rate}

Let the fixed contract payments be regularly paid in fixed percentage $\rho$ with $0 \leq \rho \leq 1$ and the outstanding debt overcharged with the late payment interests be settled through a lump-sum payment at the contract terminal date $n$ in addition to the surrender value $E \geq 0$. Let the lease contract rate $i$, the late payment rate $m$ and the contract term $n$ be exogenously given.

If the surrender value $E$ increases, then EAR goes up if the late payment rate $m$ is cheap (i.e. $m<i$ ), and $E A R$ goes down if the late payment rate $m$ is expensive (i.e. $m>i$ ).

\section{Numerical Illustrative Examples}

This section sheds light on how the payback pattern may differently impact on EAR.

Throughout this Section we assume that the leased amount is $A=150,000$, the annual compound lease contract rate is $i=5.087 \%$, the annual compound late payment rate is $m=7.719 \%$ and the installments are monthly paid.

To highlight the key drivers in determining $E A R$, we consider contracts with fixed installments and surrender value $E$, with $E \geq 0$ to be paid at the contract term. Note that we consider the case $m>i$.

To ascertain the impact of the Macaulay Duration on $E A R$ we set a number of illustrative examples. 


\section{Example 1: The impact of the surrender value on EAR}

As discussed in Result 4 a way to stick $E A R$ to contract lease rate is to prolong the Macaulay Duration $D$. If the surrender value $E$ becomes higher, the Macaulay Duration $D$ becomes longer and consequently EAR flaws down, see Table 1.

Table 1. The higher the surrender value, the longer the Duration and the smaller EAR

\begin{tabular}{ccccccc}
\hline $\begin{array}{c}\text { \# Montly } \\
\text { payments }\end{array}$ & $\begin{array}{c}\text { Fixed } \\
\text { installments }\end{array}$ & $\begin{array}{c}\text { Payment level } \\
\boldsymbol{\rho}\end{array}$ & $\begin{array}{c}\text { Surrender } \\
\text { value=\%A }\end{array}$ & $\begin{array}{c}\text { Surrender } \\
\text { value }\end{array}$ & $\begin{array}{c}\text { True annual } \\
\text { Duration }\end{array}$ & $\begin{array}{c}\boldsymbol{E A R} \\
24\end{array}$ \\
\hline 6579 & $60 \%$ & $0.00 \%$ & $\boldsymbol{0}$ & $\mathbf{1 2 . 3 0}$ & $\mathbf{5 . 8 1 2 \%}$ \\
24 & 6499 & $60 \%$ & $1.33 \%$ & $\mathbf{2 0 0 0}$ & $\mathbf{1 2 . 4 4}$ & $\mathbf{5 . 8 0 0 \%}$ \\
24 & 6420 & $60 \%$ & $2.67 \%$ & $\mathbf{4 0 0 0}$ & $\mathbf{1 2 . 5 8}$ & $\mathbf{5 . 7 8 8 \%}$ \\
24 & 6341 & $60 \%$ & $4.00 \%$ & $\mathbf{6 0 0 0}$ & $\mathbf{1 2 . 7 3}$ & $\mathbf{5 . 7 7 6 \%}$ \\
\hline
\end{tabular}

\section{Example 2: The solvency level and $E A R$}

Result 2 shows that the longer the Macauly Duration, the closer $E A R$ to the lease contract rate $i$. As intuition may suggest, if $m>i$ the lower the solvency payment level $\rho$ the higher EAR, see Table 2 .

If the payments $\rho R$ paid in due time are decreasing in size, because of $\rho$ is decreasing then $E A R$ increases and goes further from the contractual rate.

Table 2. The lower the $\rho$ the higher EAR. If the surrender value increases the EAR decreases

\begin{tabular}{lllllll}
\hline $\begin{array}{l}\text { \# Montly } \\
\text { payments }\end{array}$ & Fixed installments & $\begin{array}{l}\text { Payment } \\
\text { level } \boldsymbol{\rho}\end{array}$ & $\begin{array}{l}\text { Surrender } \\
\text { value=\%A }\end{array}$ & $\begin{array}{l}\text { Surrender } \\
\text { value }\end{array}$ & Duration & True annual $\boldsymbol{E A R}$ \\
\hline 24 & 6499 & $60 \%$ & $1.33 \%$ & 2000 & 12.44 & $5.800 \%$ \\
24 & 6499 & $40 \%$ & $1.33 \%$ & 2000 & 12.44 & $6.028 \%$ \\
24 & 6499 & $20 \%$ & $1.33 \%$ & 2000 & 12.44 & $6.207 \%$ \\
24 & 6420 & $60 \%$ & $2.67 \%$ & 4000 & 12.58 & $5.788 \%$ \\
24 & 6420 & $40 \%$ & $2.67 \%$ & 4000 & 12.58 & $6.014 \%$ \\
24 & 6420 & $20 \%$ & $2.67 \%$ & 4000 & 12.58 & $6.192 \%$ \\
24 & 6341 & $60 \%$ & $4.00 \%$ & 6000 & 12.73 & $5.776 \%$ \\
24 & 6341 & $40 \%$ & $4.00 \%$ & 6000 & 12.73 & $6.000 \%$ \\
24 & 6341 & $20 \%$ & $4.00 \%$ & 6000 & 12.73 & $6.177 \%$ \\
\hline
\end{tabular}

\section{Example 3: Accuracy of EAR numerical estimate}

Table 3. confirms the EAR approximation accuracy with respect to the true EAR value. The EAR linear approximation underestimates the true $E A R$ and the estimate $E A R$ is more accurate with increasing surrender value and the level $\rho$ of installment payments.

Table 3. Data confirm the approximation accuracy

\begin{tabular}{cccccc}
\hline Payment level $\boldsymbol{\rho}$ & Surrender value=\%A & Duration & True annual $\boldsymbol{E A R}$ & Estimated annual $\boldsymbol{E A R}$ & $\begin{array}{c}\Delta \boldsymbol{E A R} \\
\text { true - estimate }\end{array}$ \\
\hline $\mathbf{6 0} \%$ & $1.33 \%$ & 12.44 & $\mathbf{5 . 8 0 0 \%}$ & $\mathbf{5 . 7 9 6 \%}$ & $\mathbf{0 . 0 0 0 0 4 4}$ \\
$\mathbf{4 0 \%}$ & $1.33 \%$ & 12.44 & $\mathbf{6 . 0 2 8 \%}$ & $\mathbf{6 . 0 2 0 \%}$ & $\mathbf{0 . 0 0 0 0 8 0}$ \\
$\mathbf{2 0 \%}$ & $1.33 \%$ & 12.44 & $\mathbf{6 . 2 0 7 \%}$ & $\mathbf{6 . 1 9 5 \%}$ & $\mathbf{0 . 0 0 0 1 1 9}$ \\
$\mathbf{6 0 \%}$ & $2.67 \%$ & 12.58 & $\mathbf{5 . 7 8 8 \%}$ & $\mathbf{5 . 7 8 4 \%}$ & $\mathbf{0 . 0 0 0 0 4 2}$ \\
$\mathbf{4 0 \%}$ & $2.67 \%$ & 12.58 & $\mathbf{6 . 0 1 4 \%}$ & $\mathbf{6 . 0 0 6 \%}$ & $\mathbf{0 . 0 0 0 0 7 8}$ \\
$\mathbf{2 0 \%}$ & $2.67 \%$ & 12.58 & $\mathbf{6 . 1 9 2 \%}$ & $\mathbf{6 . 1 8 1 \%}$ & $\mathbf{0 . 0 0 0 1 1 6}$ \\
$\mathbf{6 0} \%$ & $4.00 \%$ & 12.73 & $\mathbf{5 . 7 7 6 \%}$ & $\mathbf{5 . 7 7 2 \%}$ & $\mathbf{0 . 0 0 0 0 4 1}$ \\
$\mathbf{4 0 \%}$ & $4.00 \%$ & 12.73 & $\mathbf{6 . 0 0 0 \%}$ & $\mathbf{5 . 9 9 2 \%}$ & $\mathbf{0 . 0 0 0 0 7 6}$ \\
$\mathbf{2 0 \%}$ & $4.00 \%$ & 12.73 & $\mathbf{6 . 1 7 7 \%}$ & $\mathbf{6 . 1 6 6 \%}$ & $\mathbf{0 . 0 0 0 1 1 3}$ \\
\hline
\end{tabular}




\section{Conclusion}

Delays in payment incur extra-costs that make the lease $E A R$ to deviate from the lease contract rate. Motivated by the practical need to design leasing contracts that keep $E A R$ under control, because of the presence in Italian and European law to kept below some interest rate caps on loans, in this paper we assume that the lease contract rate $i$, the late payment rate $m$ and the contract term be exogenously given. First we formalize the condition "the small installments come first and the large installments come later": in the event of partial insolvency, extra-costs are limited, because they are referred to small amounts, and $E A R$ remains stick to the contract lease rate $i$. Vice versa if "the large installments come first and the small installments come later" EAR diverges from the contract lease rate $i$.

We provide two general rules for the payback settlement able to make EAR close to the contract rate: (1) to set the payback settlement Macaulay Duration long; and (2) to set a large surrender value. A sufficient condition to achieve the former goal is to set the payments increasing in amount.

In the case of fixed payments, the formulae become simpler but the advices to make $E A R$ stick to the contract rate remain the same.

To make evidence of our results we roll sensitivity analysis out through numerical examples. The results of the paper are useful to provide policymakers a better knowledge about the effects of the allocation the payback amounts over the contract life.

\section{Acknowledgments}

This work was supported in part by the Department of Management under a Grant of the MIUR (Ministero dell'Istruzione, dell'Università e della Ricerca Scientifica), Italy.

\section{References}

Brealey, R. A., Myers, S., \& Allen, F. (2014). Principles of Corporate Finance (11th ed.). McGraw-Hill Irwin, New York.

Carretta, A., \& Nicolini, G. (2009). Pricing the lease during the contract: an in-progress approach to lease evaluation. Managerial Finance, 35(10), 841-859. https://doi.org/10.1108/03074350910984719

de La Grandville, O. (2003). Bond Pricing and Portfolio Analysis. Protecting Investors in the Long Run, The MIT Press Ltd, Cambridge, Mass.

Dierkes, T., \& Ortmann, K. M. (2015). On the efficient utilisation of duration, Insurance: Mathematics and Economics, 60, 29-37. https://doi.org/10.1016/j.insmatheco.2014.11.002

Macaulay, F. (1938). Some Theoretical Problems Suggested by the Movements of Interest Rates. Bond Yields and Stock Prices in the United States since 1856, New York: National Bureau of Economic Research.

Maimbo, S. M., \& Gallegos, C. A. H. (2014), Interest Rate Caps Around the World: Still Popular, But a Blunt Instrument. World Bank Policy Research Working Paper, no. 7070. Retrieved from https://ssrn.com/abstract $=2513126$

Migliavacca, A., Uberti, M., Tibiletti, L., \& Rainero, C. (2018) Financial and accounting approaches in lease appraisal, International Journal of Business and Management, 13(5), 13-20. https://doi.org/10.5539/ijbm.v13n5p13

Norstrøm, C. J. (1972). A Sufficient Condition for a Unique Nonnegative Internal Rate of Return, Journal of Financial and Quantitative Analysis, 7(3), 1835-1839. https://doi.org/10.2307/2329806

Quattrocchio, L., Tibiletti, L., \& Uberti, M. (2018). Early termination clauses for leasing contracts with APR cap, International Journal of Business and Management, 13(12), 290-305. https://doi.org/10.5539/ijbm.v13n12p290

Yoshida, J., Seko, M., \& Sumita, K. (2016). The Rent Term Premium for Cancellable Leases. The Journal of Real Estate Finance and Economics, 52(4), 480-511. https://doi.org/10.1007/s11146-015-9528-x

\section{Note}

Note 1. Due to Norstrøm (1972) condition such that a cash-flow that displays a single change in sign admits a unique positive internal rate of return, $E A R$ is the unique solution of the equation (3). In general for $n>2$ no closed formulae exist to calculate $E A R$. 


\section{Appendix A}

Considering the $N P V_{\text {late }}(x)$ of the contract including the extra-costs as a function of the annual interest rate $x$ used for discounting.

By (2) for $x=i$ we get

$$
\begin{aligned}
& N P V_{\text {late }}(i)=A-\rho \cdot \sum_{s=1}^{n} R_{s} \cdot(1+i)^{-s}-(1-\rho) \cdot\left(\frac{1+m}{1+i}\right)^{n} \cdot \sum_{s=1}^{n} R_{s} \cdot(1+m)^{-s}-\frac{E}{(1+i)^{n}}= \\
& =\left[\rho \cdot \sum_{s=1}^{n} R_{s} \cdot(1+i)^{-s}+(1-\rho) \cdot \sum_{s=1}^{n} R_{s} \cdot(1+i)^{-s}+\frac{E}{(1+i)^{n}}\right]-\rho \cdot \sum_{s=1}^{n} R_{s} \cdot(1+i)^{-s}-(1-\rho) \cdot\left(\frac{1+m}{1+i}\right)^{n} \cdot \sum_{s=1}^{n} R_{s} \cdot(1+m)^{-s}-\frac{E}{(1+i)^{n}}= \\
& =(1-\rho) \cdot\left(\sum_{s=1}^{n} R_{s} \cdot(1+i)^{-s}-\left(\frac{1+m}{1+i}\right)^{n} \cdot \sum_{s=1}^{n} R_{s} \cdot(1+m)^{-s}\right]= \\
& =(1-\rho) \cdot \frac{(1+i)^{n} \cdot \sum_{s=1}^{n} R_{s} \cdot(1+i)^{-s}-(1+m)^{n} \cdot \sum_{s=1}^{n} R_{s} \cdot(1+m)^{-s}}{(1+i)^{n}}= \\
& =(1-\rho) \cdot \frac{\sum_{s=1}^{n} R_{s} \cdot(1+i)^{n-s}-\sum_{s=1}^{n} R_{s} \cdot(1+m)^{n-s}}{(1+i)^{n}}=(1-\rho) \cdot \frac{\sum_{s=1}^{n} R_{s} \cdot\left[(1+i)^{n-s}-(1+m)^{n-s}\right]}{(1+i)^{n}}
\end{aligned}
$$

By (2) for $x=m$ and by the equation (1) it follows

$$
\begin{aligned}
& N P V_{\text {late }}(m)=A-\rho \cdot \sum_{s=1}^{n} \frac{R_{s}}{(1+m)^{s}}-(1-\rho) \cdot \sum_{s=1}^{n} \frac{R_{s}}{(1+m)^{s}}-\frac{E}{(1+m)^{n}}= \\
& =A-\sum_{s=1}^{n} \frac{R_{s}}{(1+m)^{s}}-\frac{E}{(1+m)^{n}}= \\
& =\left[\sum_{s=1}^{n} \frac{R_{s}}{(1+i)^{s}}+\frac{E}{(1+i)^{n}}\right]-\sum_{s=1}^{n} \frac{R_{s}}{(1+m)^{s}}-\frac{E}{(1+m)^{n}}= \\
& =\sum_{s=1}^{n} R_{s} \cdot\left[\frac{1}{(1+i)^{s}}-\frac{1}{(1+m)^{s}}\right]+E \cdot\left[\frac{1}{(1+i)^{n}}-\frac{1}{(1+m)^{n}}\right]
\end{aligned}
$$

Since by construction, $N P V_{\text {late }}(x)$ is a strictly increasing function in $x$ and $N P V_{\text {late }}(E A R)=0$, then

1. if $m<i$ then $N P V_{\text {late }}(i)>0$ and $N P V_{\text {late }}(m)<0$. Therefore $m<E A R<i$;

2. if $m=i$ then $N P V_{\text {late }}(i)=0$. Therefore $E A R=i$;

3. if $m>i$ then $N P V_{\text {late }}(i)<0$ and $N P V_{\text {late }}(m)>0$ Therefore $i<E A R<m$.

\section{Appendix B}

Given the annual compound lease contract rate $i$, let approximate the function (2) NPV late $(x)$ with Taylor's approximation

$$
N P V_{\text {late }}(x) \cong N P V_{\text {late }}(i)+N P V_{\text {late }}^{\prime}(i) \cdot(x-i)
$$

Let $x=E A R$. By definition $N P V_{\text {late }}(E A R)=0$ and (B.1) can be solved. For $E A R \neq i$ :

$$
E A R \cong i+\frac{-N P V_{\text {late }}(i)}{N P V_{\text {late }}^{\prime}(i)}, \text { with } N P V_{\text {late }}^{\prime}(i) \neq 0
$$


where $N P V_{\text {late }}^{\prime}(i)$ is the derivative of $N P V_{\text {late }}(x)$ with respect to $x$ at $x=i$.

- By the $N P V$ formula (2) we calculate

$$
\begin{aligned}
& N P V_{\text {late }}(i)=A-\rho \cdot \sum_{s=1}^{n} R_{s} \cdot(1+i)^{-s}-(1-\rho) \cdot\left(\frac{1+m}{1+i}\right)^{n} \cdot \sum_{s=1}^{n} R_{s} \cdot(1+m)^{-s}-\frac{E}{(1+i)^{n}}= \\
& =\left[\rho \cdot \sum_{s=1}^{n} R_{s} \cdot(1+i)^{-s}+(1-\rho) \cdot \sum_{s=1}^{n} R_{s} \cdot(1+i)^{-s}+\frac{E}{(1+i)^{n}}\right]-\rho \cdot \sum_{s=1}^{n} R_{s} \cdot(1+i)^{-s}-(1-\rho) \cdot\left(\frac{1+m}{1+i}\right)^{n} \cdot \sum_{s=1}^{n} R_{s} \cdot(1+m)^{-s}-\frac{E}{(1+i)^{n}}= \\
& =(1-\rho) \cdot\left[\sum_{s=1}^{n} R_{s} \cdot(1+i)^{-s}-\left(\frac{1+m}{1+i}\right)^{n} \cdot \sum_{s=1}^{n} \frac{R_{s}}{(1+m)^{s}}\right]= \\
& =(1-\rho) \cdot\left[A-\frac{E}{(1+i)^{n}}-\left(\frac{1+m}{1+i}\right)^{n} \cdot \sum_{s=1}^{n} \frac{R_{s}}{(1+m)^{s}}\right]=-(1-\rho) \cdot\left[\left(\frac{1+m}{1+i}\right)^{n} \cdot \sum_{s=1}^{n} \frac{R_{s}}{(1+m)^{s}}+\frac{E}{(1+i)^{n}}-A\right]= \\
& =-(1-\rho) \cdot\left(M+\frac{E}{(1+i)^{n}}-A\right)
\end{aligned}
$$

where $M=\left(\frac{1+m}{1+i}\right)^{n} \cdot \sum_{s=1}^{n} \frac{R_{s}}{(1+m)^{s}}$.

- By the $N P V$ formula (2) we calculate

$$
\begin{aligned}
& N P V_{\text {late }}^{\prime}(i)=\left.\frac{\partial N P V_{\text {late }}(x)}{\partial x}\right|_{x=i}=\rho \cdot \sum_{s=1}^{n} s \cdot R_{s}(1+i)^{-s-1}+n \cdot(1+i)^{-n-1} \cdot(1-\rho) \cdot \sum_{s=1}^{n} R_{s} \cdot(1+m)^{n-s}+n \cdot(1+i)^{-n-1} \cdot E= \\
& =\rho \cdot\left[\sum_{s=1}^{n} s \cdot R_{s}(1+i)^{-s-1}+n \cdot(1+i)^{-n-1} \cdot E\right]+n \cdot(1+i)^{-n-1} \cdot(1-\rho) \cdot\left[\sum_{s=1}^{n} R_{s} \cdot(1+m)^{n-s}+E\right]= \\
& =\rho \cdot \frac{D}{1+i} \cdot A+n \cdot(1+i)^{-n-1} \cdot(1-\rho) \cdot\left[\sum_{s=1}^{n} R_{s} \cdot(1+m)^{n-s}+E\right]= \\
& =\frac{1}{1+i} \cdot\left\{\rho \cdot D \cdot A+n \cdot(1-\rho) \cdot\left[\left(\frac{1+m}{1+i}\right)^{n} \cdot \sum_{s=1}^{n} \frac{R_{s}}{(1+m)^{s}}+\frac{E}{(1+i)^{n}}\right]\right\}= \\
& =\frac{1}{1+i} \cdot\left\{\rho \cdot D \cdot A+n \cdot(1-\rho) \cdot\left[M+\frac{E}{(1+i)^{n}}\right]\right\}
\end{aligned}
$$

where $D=\frac{\sum_{s=1}^{n} s \cdot R_{s} \cdot(1+i)^{-s}+n \cdot E \cdot(1+i)^{-n}}{A}$ is the Macaulay Duration of the contractual payback given by the payments $R_{1}, R_{2}, . ., R_{n}$ at dates $s=1, . ., n$ and the payment of surrender value $E$ at date $n$.

If extra-costs occur, then $E A R \neq i$ and formula (B.2) holds. Substituting in (B.2) the expressions of $N P V_{\text {late }}(x)$ and $N P V^{\prime}{ }_{\text {late }}(i)$ with $N P V_{\text {late }}^{\prime}(i) \neq 0$ because $N P V^{\prime}{ }_{\text {late }}(i)$ is a sum of positive addenda, we obtain

$$
E A R \cong i+\frac{-N P V_{\text {late }}(i)}{N V_{\text {late }}^{\prime}(i)}=i+(1-\rho) \cdot(1+i) \cdot \frac{\left[M+E \cdot(1+i)^{-n}\right]-A}{\rho \cdot A \cdot D+(1-\rho) \cdot\left[M+E \cdot(1+i)^{-n} \cdot n\right]}
$$

\section{Appendix C.1 The payment spread over time influence on the Macaulay Duration}

Let the contract term $n$ be exogeneously fixed and the contract payments $R_{s}$, with $s=1, \ldots, n$ and the surrender value $E$ satisfy the closure condition (1). Denote by $D$ the Macaulay Duration of the payback.

- Let us modify the payment stream $R_{s}$ with $s=1, \ldots, n$ and the surrender value $E$ be fixed.

We will show that if we move forward a portion of a payment then the Macaulay Duration becomes longer. Let $z$ be fixed, with $z=1, \ldots, n-1$. Let us assume that the payment $R_{z}$ is reduced by the amount $Q_{z}$ with 
$0<Q_{z} \leq R_{z}$. At the epoch $z+k$ where $1 \leq k \leq n-z-1$ is paid the capitalized amount $Q_{z} \cdot(1+i)^{k}$ in addition to the payment $R_{z+k}$. The modified stream

$$
R_{1}, \ldots,\left[R_{z}-Q_{z}\right], \ldots,\left[R_{z+k}+Q_{z} \cdot(1+i)^{k}\right], . ., R_{n}
$$

and the surrender value $E$ satisfy the closure condition (1), because

$$
\begin{aligned}
& \frac{R_{1}}{(1+i)^{1}}+\ldots+\frac{R_{z}-Q_{z}}{(1+i)^{z}}+\ldots+\frac{R_{z+k}+Q_{z} \cdot(1+i)^{k}}{(1+i)^{z+k}}+\ldots+\frac{R_{n}+E}{(1+i)^{n}}=\sum_{s=1}^{n} \frac{R_{s}}{(1+i)^{s}}-\frac{Q_{z}}{(1+i)^{z}}+\frac{Q_{z} \cdot(1+i)^{k}}{(1+i)^{z+k}}+\frac{E}{(1+i)^{n}}= \\
& =A-\frac{Q_{z}}{(1+i)^{z}}+\frac{Q_{z}}{(1+i)^{z}}=A
\end{aligned}
$$

The Macaulay Duration of the modified payments (C.1) becomes

$$
\begin{aligned}
& D_{(z)}=\frac{1 \cdot \frac{R_{1}}{(1+i)^{1}}+\ldots+z \cdot \frac{R_{z}-Q_{z}}{(1+i)^{z}}+\ldots+(z+k) \cdot \frac{R_{z+k}+Q_{z} \cdot(1+i)^{k}}{(1+i)^{z+k}}+\ldots+n \cdot \frac{R_{n}}{(1+i)^{n}}+n \cdot \frac{E}{(1+i)^{n}}}{A}= \\
& =\frac{\sum_{s=1}^{n} s \cdot \frac{R_{s}}{(1+i)^{s}}+n \cdot \frac{R_{n}}{(1+i)^{n}}+\frac{Q_{z}}{(1+i)^{z}} \cdot(-z+z+k)}{A}=D+k \cdot \frac{Q_{z} \cdot(1+i)^{-z}}{A}
\end{aligned}
$$

It is immediate to check that $D_{(z)}$ is an increasing function of the $k$ period payment postponement of the amount $Q_{z}$. The same reasoning can be rolled out by modifying simultaneously more than one payment. $A$ fortiori the Macaulay Duration increases.

- Let $E^{\prime}$ be the surrender value with $E^{\prime}>E$.

The modified payment stream $R_{s}^{\prime}$, with $s=1, \ldots, n$ must satisfy the closure condition (1)

$$
A=\sum_{s=1}^{n} \frac{R_{s}^{\prime}}{(1+i)^{s}}+\frac{E^{\prime}}{(1+i)^{n}}
$$

It follows that there exists at least one $z$, such that $R_{z}^{\prime}<R_{z}$ and $E^{\prime}=E+\left(R_{s}-R_{z}^{\prime}\right) \cdot(1+i)^{n-z}$ with $R_{z}^{\prime}=R_{z}$ with $s \neq z$. Denoted by $D^{\prime}$ the Macaulay Duration of the payment stream $R_{s}^{\prime}$ with $s=1, \ldots, n$, it results $D^{\prime}>D$.

In conclusion the more postponed the payments, the longer the Macaulay Duration; and the sooner the payments, the shorter the Macaulay Duration.

\section{Appendix C.2 The influence of Macaulay Duration on $E A R$}

Let us calculate (3.1.1) for the modified stream in (C.1):

$$
\begin{aligned}
& M_{(z)}=\left(\frac{1+m}{1+i}\right)^{n} \cdot\left[\frac{R_{1}}{(1+m)^{1}}+\ldots+\frac{R_{z}-Q_{z}}{(1+m)^{z}}+\ldots+\frac{R_{z+k}+Q_{z} \cdot(1+i)^{k}}{(1+m)^{z+k}}+\ldots+\frac{R_{n}}{(1+m)^{n}}\right]= \\
& =M+\left(\frac{1+m}{1+i}\right)^{n} \cdot\left[\frac{-Q_{z}}{(1+m)^{z}}+\frac{Q_{z} \cdot(1+i)^{k}}{(1+m)^{z+k}}\right]=M+\left(\frac{1+m}{1+i}\right)^{n} \cdot Q_{z} \cdot\left[\frac{(1+i)^{k}-(1+m)^{k}}{(1+m)^{z+k}}\right]
\end{aligned}
$$

By construction, $D_{(z)}$ in (C.2) is such that $D_{(z)}>D$, and 
a) if $m<i$, then $M_{(z)}>M$; then the larger $M_{(z)}$, the longer the Macaulay Duration $D_{(z)}$ and the function $D=D(M)$ is increasing;

b) if $i<m$, then $M_{(z)}<M$; then the larger $M_{(z)}$, the shorter the Macaulay Duration $D_{(z)}$, therefore the function $D=D(M)$ is decreasing.

The extra-charge addendum defined in (3.1) is given by

$$
\varepsilon=(1+i) \cdot(1-\rho) \cdot \frac{\left[M+E \cdot(1+i)^{-n}\right]-A}{\rho \cdot A \cdot D+(1-\rho) \cdot\left[M+E \cdot(1+i)^{-n}\right] \cdot n}
$$

Let us calculate

$$
\begin{aligned}
& \frac{\partial \varepsilon}{\partial M}= \\
& =(1+i) \cdot(1-\rho) \cdot \frac{D \cdot \rho \cdot A+n \cdot(1-\rho) \cdot\left[M+E \cdot(1+i)^{-n}\right]-\left[M+E \cdot(1+i)^{-n}-A\right] \cdot\left[\frac{\partial D}{\partial M} \cdot \rho \cdot A+n \cdot(1-\rho)\right]}{\left\{\rho \cdot A \cdot D+(1-\rho) \cdot\left[M+E \cdot(1+i)^{-n}\right] \cdot n\right\}^{2}}= \\
& =(1+i) \cdot(1-\rho) \cdot \frac{D \cdot \rho \cdot A+n \cdot(1-\rho) \cdot A-\frac{\partial D}{\partial M} \cdot \rho \cdot A \cdot\left[M+E \cdot(1+i)^{-n}-A\right]}{\left\{\rho \cdot A \cdot D+(1-\rho) \cdot\left[M+E \cdot(1+i)^{-n}\right] \cdot n\right\}^{2}}
\end{aligned}
$$

Then

a) if $m<i$, due to Remark 1 and formulae (4), $\varepsilon<0$; therefore $\left[M+E \cdot(1+i)^{-n}\right]-A<0$. Since $\frac{\partial M}{\partial D} \geq 0$, the numerator of (C.3) is non negative and $\frac{\partial \varepsilon}{\partial M} \geq 0 ;$

b) if $m=i$, due to Remark $1, \varepsilon=0$;

c) if $i<m$, due to Remark 1 and formulae (4), $\varepsilon>0$; therefore $\left[M+E \cdot(1+i)^{-n}\right]-A>0$. Since $\frac{\partial M}{\partial D} \leq 0$, the numerator of $(\mathrm{C} .3)$ is non negative and $\frac{\partial \varepsilon}{\partial M} \geq 0$.

Then

a) if $m<i$, the longer the Macaulay Duration $D$, the larger $M$, the greater $\varepsilon$. Then the longer the Macaulay Duration $D$, the more $E A R$ is closer to $i$;

b) if $m=i$, then $E A R=i$;

c) if $i<m$, the longer the Macaulay Duration $D$, the smaller $M$, the smaller $\varepsilon$. Then the longer the Macaulay Duration $D$, the more EAR is closer to $i$.

We conclude that the longer the Macaulay Duration, the shorter $|\varepsilon|$ and the closer $E A R$ to the contract rate $i$. Vice versa, the shorter the Macaulay Duration, the longer $|\varepsilon|$ and the more EAR goes to its extreme values.

\section{Appendix D Fixed instalements: the Macaulay Duration}

The Macaulay Duration of a level annuity with zero-price surrender value is 


$$
D_{E=0}=1+\frac{1}{i}-\frac{n}{(1+i)^{n}-1}
$$

see Dierkes and Ortmann (2015). Note that $D_{E=0}$ is independent of the amount $R$ of the fixed instalment and the leased amount $A$.

The Macaulay Duration of the payment stream in the presence of $E$ with $E \geq 0$ becomes

$$
\begin{aligned}
& D=\frac{\sum_{s=1}^{n} s \cdot R_{E} \cdot(1+i)^{-s}+n \cdot E \cdot(1+i)^{-n}}{A}=\frac{\left[R-\frac{E \cdot(1+i)^{-n}}{a_{\tilde{n} \mid}}\right] \cdot \sum_{s=1}^{n} s \cdot(1+i)^{-s}+n \cdot E \cdot(1+i)^{-n}}{A}= \\
& =\frac{R \cdot \sum_{s=1}^{n} s \cdot(1+i)^{-s}-E \cdot(1+i)^{-n} \cdot \frac{\sum_{s=1}^{n} s \cdot(1+i)^{-s}}{a_{\pi \mid i}}+n \cdot E \cdot(1+i)^{-n}}{A}= \\
& =\frac{R \cdot \sum_{s=1}^{n} s \cdot(1+i)^{-s}}{A}-\frac{E \cdot(1+i)^{-n}}{A} \cdot D_{E=0}+n \cdot \frac{E \cdot(1+i)^{-n}}{A}= \\
& =D_{E=0}+\frac{E}{A} \cdot(1+i)^{-n} \cdot\left(n-D_{E=0}\right)
\end{aligned}
$$

Therefore,

- if $E=0$ then $D=D_{E=0}$; and

- if $E>0$ then $D>D_{E=0}$.

\section{Appendix E. Fixed installments: $E A R$}

The extra-charge rate addendum is given by (3.2):

$$
\begin{aligned}
& \varepsilon=(1+i) \cdot(1-\rho) \cdot \frac{\left[M+\frac{E}{(1+i)^{n}}\right]-A}{\rho \cdot A \cdot D+(1-\rho) \cdot\left[M+\frac{E}{(1+i)^{n}}\right] \cdot n}= \\
& =(1-\rho) \cdot(1+i) \cdot \frac{\left[R_{E} \cdot\left(\frac{1+m}{1+i}\right)^{n} \cdot a_{n \mid m}+\frac{E}{(1+i)^{n}}\right]-\left[R_{E} \cdot a_{n \mid i}+\frac{E}{(1+i)^{n}}\right]}{\rho \cdot\left[R_{E} \cdot a_{n \mid i}+\frac{E}{(1+i)^{n}}\right] \cdot D+(1-\rho) \cdot\left[R_{E} \cdot\left(\frac{1+m}{1+i}\right)^{n} \cdot a_{n \mid m}+\frac{E}{(1+i)^{n}}\right] \cdot n}= \\
& =(1-\rho) \cdot(1+i) \cdot \frac{R_{E} \cdot\left[\left(\frac{1+m}{1+i}\right)^{n} \cdot a_{n \mid m}-a_{n \mid i}\right]}{\rho \cdot\left[R_{E} \cdot a_{n \mid i}+\frac{E}{(1+i)^{n}}\right] \cdot D+(1-\rho) \cdot\left[R_{E} \cdot\left(\frac{1+m}{1+i}\right)^{n} \cdot a_{n \mid m}+\frac{E}{(1+i)^{n}}\right] \cdot n}= \\
& =(1-\rho) \cdot(1+i) \cdot \frac{R_{E} \cdot\left[(1+m)^{n} \cdot a_{n \mid m}-(1+i)^{n} \cdot a_{n \mid i}\right]}{\rho \cdot\left[R_{E} \cdot(1+i)^{n} \cdot a_{n \mid i}+E\right] \cdot D+(1-\rho) \cdot\left[R_{E} \cdot(1+m)^{n} \cdot a_{n \mid m}+E\right] \cdot n}= \\
& =(1-\rho) \cdot(1+i) \cdot \frac{R_{E} \cdot\left[s_{n \mid m}-s_{n \mid i}\right]}{\rho \cdot\left[R_{E} \cdot s_{n \mid i}+E\right] \cdot D+(1-\rho) \cdot\left[R_{E} \cdot s_{n \mid m}+E\right] \cdot n}= \\
& =(1-\rho) \cdot(1+i) \cdot \frac{R_{E} \cdot\left[\frac{s_{n \mid m}}{s_{n \mid i}}-1\right]}{\rho \cdot\left[R_{E}+\frac{E}{s_{n \mid i}}\right] \cdot D+(1-\rho) \cdot\left[R_{E} \cdot \frac{s_{n \mid m}}{s_{n \mid i}}+\frac{E}{s_{n \mid i}}\right] \cdot n}
\end{aligned}
$$

\section{Copyrights}

Copyright for this article is retained by the author(s), with first publication rights granted to the journal.

This is an open-access article distributed under the terms and conditions of the Creative Commons Attribution license (http://creativecommons.org/licenses/by/4.0/). 